\title{
Ultrafast spin dynamics across compensation points in ferrimagnetic GdFeCo: The role of angular momentum compensation
}

\author{
C. D. Stanciu, ${ }^{1}$ A. V. Kimel, ${ }^{1}$ F. Hansteen, ${ }^{1}$ A. Tsukamoto, ${ }^{1,2}$ A. Itoh, ${ }^{2}$ A. Kirilyuk,,${ }^{1}$ and Th. Rasing ${ }^{1}$ \\ ${ }_{1}^{1}$ Institute for Molecules and Materials, Radboud University Nijmegen, Toernooiveld 1, 6525 ED Nijmegen, The Netherlands \\ ${ }^{2}$ College of Science and Technology, Nihon University, 7-24-1 Funabashi, Chiba, Japan
}

(Received 15 May 2006; published 12 June 2006)

\begin{abstract}
Using an all-optical pump and probe technique, we have investigated the temperature dependence of the ultrafast magnetic response in a ferrimagnetic amorphous GdFeCo thin film. When the temperature of the sample approaches the angular momentum compensation point, both frequency and the Gilbert damping parameter of the magnetization precession increase significantly. In addition, the high-frequency exchange mode softens and becomes observable. The observed high-speed and strongly damped spin dynamics in the vicinity of the compensation of the angular momentum is ideal for ultrafast ringing-free precessional switching in magnetic and magneto-optical recording.
\end{abstract}

DOI: 10.1103/PhysRevB.73.220402

PACS number(s): 75.50.Gg, 78.47.+p, 75.50.Ss, 76.50.+g

The study of ultrafast magnetization dynamics has gained considerable momentum in the last decade. This is stimulated on the one hand by the ever growing demands to increase the speed of writing and retrieving magnetically stored information, and on the other, by the development of femtosecond laser sources. The latter allow excitation of magnetic systems at time scales much shorter than fundamental quantities such as spin precession or spin-lattice relaxation times. Recent experimental studies have revealed many exciting and sometimes surprising results such as the quenching of ferromagnetism at subpicosecond time scales, ${ }^{1-3}$ generation of coherent spin waves, ${ }^{4,5}$ and reorientation of antiferromagnetic spins. ${ }^{6}$

Rare earth-3d transition metal (RE-TM) ferrimagnetic compounds are widely used materials for magneto-optical recording. Depending on their composition, RE-TM ferrimagnets can exhibit a magnetization compensation temperature $T_{M}$ where the magnetizations of the RE and TM sublattices cancel each other and similarly, an angular momentum compensation temperature $T_{A}$ where the net angular momentum of the sublattices vanishes. The theory of ferrimagnetic resonance $^{7,8}$ predicts a strong temperature dependence of the dynamic behavior in such systems. In particular, the frequency of the homogeneous spin precession as well as the Gilbert damping parameter $\alpha$ are expected to diverge at the temperature $T_{A} .{ }^{9}$ However, so far there are no experimental results to substantiate these claims. Experimental confirmation of these theoretical predictions is important for magnetic recording and magnetic random access memory, since the combination of a high frequency and large damping of the spin precession would provide ultrafast and ringing-free magnetization reversal via precessional motion. ${ }^{10}$ In addition, knowledge of the temperature behavior of the frequency and damping of the spin precession is crucial for the calculation of the domain wall velocity ${ }^{11}$ and thus of great importance for magneto-optical recording. Although of fundamental and technological interest, the temperature dependence of the Gilbert damping and of the magnetization precession frequency over the compensations points in multisublattice magnetic systems such as ferrimagnetic RE-TM alloys were not well known until now.
In this Rapid Communication we report the study of ultrafast laser-induced spin dynamics in the RE-TM ferrimagnetic alloy $\mathrm{GdFeCo}$ across the magnetization and the angular momentum compensation points, using an all-optical pumpprobe technique. It is demonstrated experimentally that both Gilbert damping and spin precession frequency increase significantly when the temperature of the sample approaches the point of angular momentum compensation. These results indicate thus the crucial role of the angular momentum compensation for controlling the fast magnetization switching process. Moreover, our approach also allowed us to observe the exchange resonance mode: as it softens near $T_{A}$ it is found to dominate the ordinary ferromagnetic resonance.

The sample used in our experiments was a 20 -nm-thick GdFeCo layer, such as used in a magnetically amplified magneto-optical system (MAMMOS) ${ }^{12}$ as a readout layer. The total multilayer consisting of glass/AlTi(10 nm)/ $\mathrm{SiN}(5 \mathrm{~nm}) / \mathrm{GdFeCo}(20 \mathrm{~nm}) / \operatorname{SiN}(60 \mathrm{~nm})$, was grown by magnetron sputtering. The AlTi layer served as a heat sink. The GdFeCo layer is a ferrimagnetic amorphous alloy with strong perpendicular magnetic anisotropy. Strong coupling between the RE and TM subsystems leads to a common $\mathrm{Cu}$ rie temperature $\left(T_{C}\right)$ which for the given alloy composition $\left(\mathrm{Gd}_{22} \mathrm{Fe}_{74.6} \mathrm{Co}_{3.4}\right)$ is about $500 \mathrm{~K}$.

The dynamics of the macroscopic magnetization $\mathbf{M}$ is routinely described by the Landau-Lifshitz-Gilbert (LLG) equation. ${ }^{13,14}$ In a ferrimagnetic system, the LLG equation can be written for the $i$ th sublattice $(i=\mathrm{RE}, \mathrm{TM})$ as

$$
\frac{d \mathbf{M}_{i}}{d t}=-\left|\gamma_{i}\right|\left(\mathbf{M}_{i} \times \mathbf{H}_{i}^{\mathrm{eff}}\right)+\frac{\alpha_{i}}{M_{i}}\left(\mathbf{M}_{i} \times \frac{d \mathbf{M}_{i}}{d t}\right),
$$

with the gyromagnetic ratio $\left|\gamma_{i}\right|$ and the Gilbert damping parameter $\alpha_{i}$ given by

$$
\left|\gamma_{i}\right|=g_{i} \frac{\mu_{B}}{\hbar}, \quad \alpha_{i}=\frac{\lambda_{i}}{\left|\gamma_{i}\right| M_{i}} .
$$

Here $\lambda$ is the Landau-Lifshitz damping parameter. ${ }^{13}$ These equations are coupled by the presence of the exchange field $\mathbf{H}_{\mathrm{RE}, \mathrm{TM}}^{\mathrm{ex}}=-\lambda_{\mathrm{ex}} \mathbf{M}_{\mathrm{TM}, \mathrm{RE}}$ and give rise to two modes. The ferromagnetic mode with $\omega_{\mathrm{FMR}}=\gamma_{\mathrm{eff}} \mathbf{H}^{\text {eff }}$ can actually be described 
by a single LLG equation, but now employing an effective gyromagnetic ratio $\gamma_{\text {eff }} \cdot{ }^{7,15}$

$$
\gamma_{\mathrm{eff}}(T)=\frac{M_{\mathrm{RE}}(T)-M_{\mathrm{TM}}(T)}{\frac{M_{\mathrm{RE}}(T)}{\left|\gamma_{\mathrm{RE}}\right|}-\frac{M_{\mathrm{TM}}(T)}{\left|\gamma_{\mathrm{TM}}\right|}}=\frac{M(T)}{A(T)}
$$

and an effective Gilbert damping parameter ${ }^{16} \alpha_{\text {eff }}$

$$
\alpha_{\mathrm{eff}}(T)=\frac{\frac{\lambda_{\mathrm{RE}}}{\left|\gamma_{\mathrm{RE}}\right|^{2}}+\frac{\lambda_{\mathrm{TM}}}{\left|\gamma_{\mathrm{TM}}\right|^{2}}}{\frac{M_{\mathrm{RE}}(T)}{\left|\gamma_{\mathrm{RE}}\right|}-\frac{M_{\mathrm{TM}}(T)}{\left|\gamma_{\mathrm{TM}}\right|}}=\frac{A_{0}}{A(T)}
$$

where $M(T)$ and $A(T)$ are the net magnetic moment and net angular momentum, respectively; $A_{0}$ is a constant under the assumption of the Landau-Lifshitz damping parameter being independent of temperature. ${ }^{9}$ The validity of this assumption was confirmed over a wide temperature interval by ferromagnetic resonance (FMR) measurements in $3 d$ TMs. ${ }^{17}$ Because $\mathrm{GdFeCo}$ contains $S$-state RE ions and orbital-momentumquenched $3 d$ ions, it is expected to possess equivalent gyromagnetic ratios for the two sublattices $\left(\gamma_{\mathrm{RE}}=\gamma_{\mathrm{TM}}\right)$. However, previous experiments on similar RE-TM (i.e., Gd-Fe) thin films ${ }^{18}$ and the present experiments reveal a clear temperature dependence of $\gamma_{\mathrm{eff}}$, thus indicating that the assumption $\gamma_{\mathrm{RE}}=\gamma_{\mathrm{TM}}$ in $\mathrm{GdFeCo}$ does not hold.

In addition to the ferromagnetic mode $\omega_{\mathrm{FMR}}$, spins in a ferrimagnetic system may oscillate with the exchange (Kaplan and Kittel) resonance frequency ${ }^{19}$

$$
\omega_{\mathrm{ex}}=\lambda_{\mathrm{ex}}\left(\left|\gamma_{\mathrm{TM}}\right| M_{\mathrm{RE}}-\left|\gamma_{\mathrm{RE}}\right| M_{\mathrm{TM}}\right)=\lambda_{\mathrm{ex}}\left|\gamma_{\mathrm{RE}}\right|\left|\gamma_{\mathrm{TM}}\right| A(T) \text {. }
$$

Equations (3) and (4) indicate a divergence of both the precession frequency and Gilbert damping parameter of the FMR mode at the temperature $T_{A}$. Moreover, from Eq. (3), one can notice that at the temperature $T_{M}$, the FMR frequency becomes zero. In contrast, Eq. (5) indicates that the exchange resonance branch softens at the angular momentum compensation temperature $T_{A},{ }^{20}$ where the FMR mode diverges.

The laser-induced spin dynamics was investigated by an all-optical pump and probe technique using an amplified Ti:sapphire laser system with 100 fs laser pulses of $1.54 \mathrm{eV}$ photon energy (805 $\mathrm{nm}$ laser wavelength) at a repetition rate of $1 \mathrm{kHz}$. The measurements were done in a cold finger cryostat where the temperature could be stabilized in the range of $10-400 \mathrm{~K}$ with a precision better than $0.5 \mathrm{~K}$. The magnetization response of the almost instantaneous heating by the pump beam was probed by a less intense probe beam $\left(I_{\text {pump }} / I_{\text {probe }}>100\right)$, which monitored in time the changes in the Faraday rotation with the help of a balanced diode technique.

In our experiments, an external field $\mathbf{H}_{\mathrm{ext}}$, applied to the sample at an angle $\theta_{\mathrm{ext}}=60^{\circ}$ from the easy axis of anisotropy, tilts the magnetization $\mathbf{M}$ along the direction of an effective field $\mathbf{H}^{\text {eff }}=\mathbf{H}_{\mathrm{ext}}+\mathbf{H}_{a}+\mathbf{H}_{s}$, where $\mathbf{H}_{a}$ and $\mathbf{H}_{s}$ are the magnetocrystalline and shape anisotropy fields, respectively. Upon sudden heating by the pump pulse, $\mathbf{H}_{s}$ and $\mathbf{H}_{a}$ are changed.

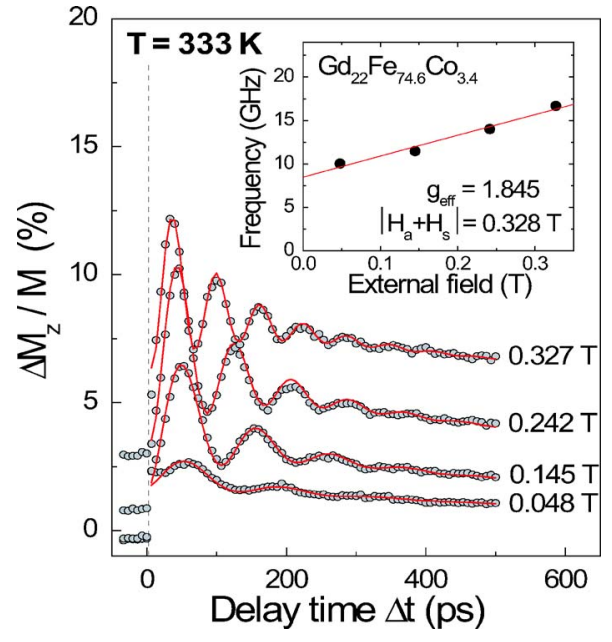

FIG. 1. (Color online) Magnetization precession in GdFeCo as a function of the applied external field at $T=333 \mathrm{~K}$. Solid lines in the main figure represent fit of the data to the LLG equation with $\alpha_{\text {eff }}=0.11$. Inset shows the field dependence of the precession frequency. The solid line is a fit to Eq. (6). Note that the sample temperature presented here includes the temperature increase induced by the pump heating (Ref. 22).

Due to the change of $\mathbf{H}^{\text {eff }}, \mathbf{M}$ starts to precess around the new equilibrium orientation. After several hundred picoseconds, electron-phonon dissipation processes remove the excess heat from the system, restoring the initial equilibrium and magnitude of $\mathbf{M}$. In the present configuration, the experiment probes changes in the out-of-plane component of the magnetization $M_{z}$. Note that at a photon energy of $1.54 \mathrm{eV}$ the main contribution to the observed component of the magnetization $M_{z}$ is given by the magnetization of the TM sub$\operatorname{system}\left(\mathbf{M}_{\mathrm{TM}}\right)^{21}$

Figure 1 shows the observed precession excited at a pump fluence of about $2 \mathrm{~mJ} / \mathrm{cm}^{2}$. This pump fluence induces a significant increase in the local temperature of the sample. ${ }^{22}$ The temperature $T=333 \mathrm{~K}$ indicated in the figure takes into account this heating. Besides the oscillatory character of the $M_{z}$ component of the magnetization associated with magnetization precession, Fig. 1 shows also an instantaneous steplike change of $M_{z}$ following laser excitation. The observed steplike process is a result of both the ultrafast demagnetization ( $\sim 200 \mathrm{fs})$ and the laser-induced change in the magnetocrystalline anisotropy which occurs while the electron-lattice equilibration take place $(\sim 3 \mathrm{ps}) .^{5,23}$ Fitting the experimental data yields precession frequencies of several gigahertz and a common Gilbert damping $\alpha_{\text {eff }}=0.11$, in good agreement with FMR results. ${ }^{24}$ The precession frequencies are well fitted by the Kittel equation which, for the geometry of interest here, is

$$
\omega=\frac{\gamma_{\mathrm{eff}}}{\left(1+\alpha_{\mathrm{eff}}^{2}\right)} \sqrt{\left(H_{\mathrm{ext}} \cos \theta_{\mathrm{ext}}+\mid H_{\mathrm{a}}+H_{\mathrm{s}}\right)^{2}-\left(H_{\mathrm{ext}} \sin \theta_{\mathrm{ext}}\right)^{2}} .
$$

Thus the measurements representing $T=333 \mathrm{~K}$ are well described by an internal field $\left|H_{a}+H_{s}\right|=0.328 \mathrm{~T}$ and an effec- 


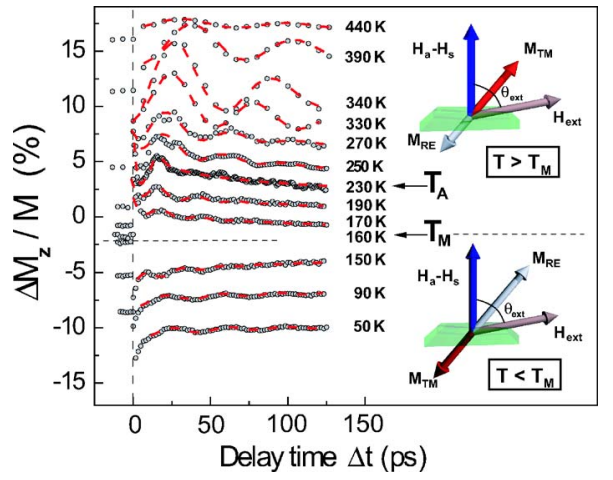

FIG. 2. (Color online) Temperature dependence of coherent precession of the magnetization in $\mathrm{GdFeCo}$, measured at an external field $\mathbf{H}_{\text {ext }}=0.29 \mathrm{~T}$. Around $160 \mathrm{~K}$ magnetic compensation $T_{M}$ of the ferrimagnetic system occurs. The inset shows the alignment of the RE-TM system under an external applied field, below and above $T_{M}$.

tive $g$ factor $g_{\text {eff }}=1.845$, which is in very good agreement with previous measurement ${ }^{25}$ on $\mathrm{GdFeCo}\left(g_{\text {eff }}=1.85\right)$. This observation indicates on the one hand that the effective gyromagnetic ratio $\gamma_{\mathrm{eff}}$ is dominated by the spin part of the angular momentum in the GdFeCo case and on the other, that at $333 \mathrm{~K}$ only the FMR branch is observed.

The temperature dependence of the magnetization dynamics at an external field $\mathbf{H}_{\text {ext }}=0.29 \mathrm{~T}$ is shown in Fig. 2. Apart from a clear temperature dependence of the frequency and damping, Fig. 2 shows that the phase of the oscillations changes around $160 \mathrm{~K}$. This happens because of the presence of the compensation point $T_{M}$. For $T<T_{M}(\sim 160 \mathrm{~K})$ the TM magnetic moment is smaller than that of $\mathrm{Gd}$. Thus the $\mathbf{M}_{\mathrm{RE}}$ is aligned along the applied field direction. At $T>T_{M}, \mathbf{M}_{\mathrm{TM}}$ becomes larger than $\mathbf{M}_{\mathrm{RE}}$ and the ferrimagnetic system flips, allowing the TM component to be aligned along the direction of the applied field $\mathbf{H}_{\text {ext }}$. It is clear that this will lead to a sign change of the observed component of the magnetization, $\mathbf{M}_{\mathrm{TM}}$.

Figure 3 shows the temperature dependence of the magnetization precession frequency (a) and the Gilbert damping parameter (b). At the temperature $T=220 \mathrm{~K}$ a significant increase is observed in both the precession frequency and the damping parameter. As expected from Eqs. (3) and (4), the fact that both $\alpha_{\text {eff }}$ and $\omega_{\text {FMR }}$ peak at the same temperature, clearly indicates the existence of angular momentum compensation near this temperature of $220 \mathrm{~K}$. The strong temperature dependence of $\gamma_{\mathrm{eff}}$ demonstrates the nonequivalent character of the gyromagnetic ratios of the two magnetic sublattices in GdFeCo. In addition to the peak near $T_{A}$, we have also observed an enhancement of $\alpha_{\text {eff }}$ as the temperature is increased toward the Curie temperature. Again Eq. (4) predicts this enhancement under the assumption of temperature-independent Landau-Lifshitz damping parameters $\lambda_{\mathrm{RE}}$ and $\lambda_{\mathrm{TM}}$. This enhancement is consistent with earlier data. ${ }^{26,27}$ Our measurements thus demonstrate the consistency of the theoretical prediction of Eq. (4) with the temperature dependence of $\alpha_{\text {eff }}$ in RE-TM alloys like GdFeCo.

In the temperature region just above $T_{A}$, Fourier analysis

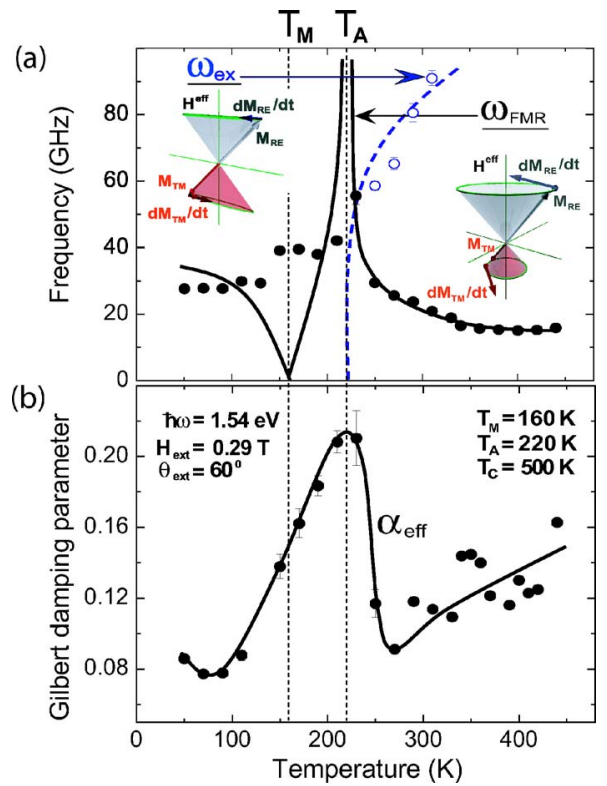

FIG. 3. (Color online) (a) Temperature dependence of the magnetization precession frequencies $\omega_{\mathrm{FMR}}$ and $\omega_{\mathrm{ex}}$. As temperature decreases from $310 \mathrm{~K}$ toward $T_{A}$, the exchange resonance mode $\omega_{\mathrm{ex}}$ (open circles) softens and mix with the ordinary FMR resonance $\omega_{\text {FMR }}$ (closed circles). Since around $230 \mathrm{~K}$ both FMR and exchange modes have essentially the same frequency, the frequency indicated at $230 \mathrm{~K}$ may represent both the FMR and the exchange resonance modes. The insets show schematically the two modes. The solid lines are a qualitative representation of the expected trend of the two resonance branches as indicated by Eqs. (3) and (5). (b) Temperature dependence of the Gilbert damping parameter $\alpha_{\text {eff }}$ Lines are guides to the eye.

of the measured time dependencies reveals two frequencies, one decreasing and the other increasing with temperature. While the former can be attributed to the FMR mode (see Fig. 1), such temperature behavior allows one to clearly identify the latter as the exchange mode [see Eq. (5)], the frequency of which can be low around $T_{A}$ but is usually very high everywhere else.

Although around $T_{A}$ the temperature dependence of the precession frequency appears to be in very good agreement with theory, this is not the case for the region near $T_{M}$. From Eq. (3) one would expect a strong decrease of the precession frequency at $T_{M}$. Instead, at this temperature Fig. 3(a) indicates still values of tens of gigahertz for the precession frequency. The reason for this apparent disagreement can be twofold. First of all, in the laser excitation spot, an inhomogeneity of the instantaneous temperature distribution is unavoidable. Therefore, when excited with a laser pulse in such a strong temperature dependence region as near $T_{M}$, a whole spectrum of frequencies can be excited with the signals from various modes adding up. It is easy to show, however, that such averaging favors observation of the highest frequency only, i.e.,

$$
\int_{0}^{\omega_{0}} \cos \omega t d \omega=\frac{1}{t} \sin \omega_{0} t .
$$

Second, in this region close to the angular momentum compensation point, the exchange mode softens. Mixing of the 
latter with the regular FMR mode can also result in the increase of the observable frequency.

In conclusion, using magneto-optical techniques we have investigated spin dynamics in ferrimagnetic GdFeCo films with subpicosecond temporal resolution and in a broad temperature range. Our data unambiguously reveal the angular momentum compensation around $T=220 \mathrm{~K}$ and magnetization compensation around $T=160 \mathrm{~K}$. The observed difference between the points of magnetization and angular momentum compensation indicates the fact that the two magnetic sublattices in the studied GdFeCo alloy have different $g$ factors. We have experimentally observed both ferromagnetic and exchange modes of spin precession in the ferrimagnetic material. While the precession frequency and the Gilbert damping strongly increase when the temperature approaches $T_{A}$, the high-frequency exchange mode softens near the angular momentum compensation point $T_{A}$, so that ferromagnetic and exchange modes have similar frequencies. This similarity may result in an additional mechanism for increase of the Gilbert damping. The observed high-speed and strongly damped spin dynamics in the vicinity of the compensation of the angular momentum is ideal for ultrafast ringing-free precessional switching in magnetic and magneto-optical recording.

The authors acknowledge the financial support of the RTN network DYNAMICS, NWO, FOM, and a Grant-inAid for Scientific Research of the Ministry of Education, Culture, Sports, Science and Technology in Japan, Grant No. 16360182.
${ }^{1}$ E. Beaurepaire, J.-C. Merle, A. Daunois, and J.-Y. Bigot, Phys. Rev. Lett. 76, 4250 (1996).

${ }^{2}$ A. Scholl, L. Baumgarten, R. Jacquemin, and W. Eberhardt, Phys. Rev. Lett. 79, 5146 (1997).

${ }^{3}$ J. Hohlfeld, E. Matthias, R. Knorren, and K. H. Bennemann, Phys. Rev. Lett. 78, 4861 (1997).

${ }^{4}$ G. Ju, A. V. Nurmikko, R. F. C. Farrow, R. F. Marks, M. J. Carey, and B. A. Gurney, Phys. Rev. Lett. 82, 3705 (1999).

${ }^{5}$ M. van Kampen, C. Jozsa, J. T. Kohlhepp, P. LeClair, L. Lagae, W. J. M. de Jonge, and B. Koopmans, Phys. Rev. Lett. 88, 227201 (2002).

${ }^{6}$ A. V. Kimel, A. Kirilyuk, A. Tsvetkov, R. V. Pisarev, and Th. Rasing, Nature (London) 429, 850 (2004).

${ }^{7}$ R. K. Wangsness, Phys. Rev. 91, 1085 (1953).

${ }^{8}$ R. K. Wangsness, Phys. Rev. 93, 68 (1954).

${ }^{9}$ T. Kobayashi, H. Hayashi, Y. Fujiwara, and S. Shiomi, Proceedings, INTERMAG 2005 (unpublished).

${ }^{10}$ H. W. Schumacher, C. Chappert, P. Crozat, R. C. Sousa, P. P. Freitas, J. Miltat, J. Fassbender, and B. Hillebrands, Phys. Rev. Lett. 90, 017201 (2003).

${ }^{11}$ Robert S. Weng and Mark H. Kryder, IEEE Trans. Magn. 29, 2177 (1993).

${ }^{12}$ H. Awano, S. Ohnuki, H. Shirai, N. Ohta, A. Yamaguchi, S. Sumi, and K. Torazawa, Appl. Phys. Lett. 69 (27), 4257 (1996).

${ }^{13}$ L. Landau and E. Lifshitz, Phys. Z. Sowjetunion 8, 153 (1935).
${ }^{14}$ T. L. Gilbert, Phys. Rev. 100, 1243 (1955).

${ }^{15}$ C. Kittel, Phys. Rev. 76, 743 (1949).

${ }^{16}$ M. Mansuripur, The Physical Principles of Magneto-Optical Recording (Cambridge University Press, Cambridge, U.K., 1995).

${ }^{17}$ S. M. Bhagat and P. Lubitz, Phys. Rev. B 10, 179 (1974).

${ }^{18}$ P. Lubitz, J. Schelleng, and C. Vittoria, Solid State Commun. 18, 965 (1976).

${ }^{19}$ J. Kaplan and C. Kittel, J. Chem. Phys. 21, 760 (1953).

${ }^{20}$ R. K. Wangsness, Phys. Rev. 97, 831 (1955).

${ }^{21}$ T. Katayama, M. Miyazaki, H. Arimune, and T. Shibata, J. Magn. Soc. Jpn. 8, 121 (1984).

${ }^{22}$ To estimate the increase in local sample temperature induced by the pump pulses we have performed static measurements. An increase in temperature of about $40 \mathrm{~K}$ was observed. The temperatures represented in Figs. 1-3 were modified accordingly.

${ }^{23}$ M. Vomir, L. H. F. Andrade, L. Guidoni, E. Beaurepaire, and J.-Y. Bigot, Phys. Rev. Lett. 94, 237601 (2005).

${ }^{24}$ A. G. Gurevich and G. A. Melkov, Magnetization Oscillations and Waves (CRC Press, Boca Raton, FL, 1996).

${ }^{25}$ A. Tsukamoto, K. Nakagawa, A. Itoh, A. Kimel, A. Tsvetkov, H. Awano, N. Ohta, A. Kirilyuk, and Th. Rasing, J. Magn. Soc. Jpn. 28, 318 (2004).

${ }^{26}$ T. Silva, J. Nibarger, B. Rippard, and B. Stamps (unpublished).

${ }^{27}$ Yi Li and K. Baberschke, Phys. Rev. Lett. 68, 1208 (1992). 